\title{
Recurrence in Intracranial Atherosclerotic Disease: A Stenosis-based Analysis
}

\author{
Ana Gouveia, MD, ${ }^{*}$ João Sargento-Freitas, MD, + Joana Penetra, MD, $\neq$ \\ Fernando Silva, MD, + Cristina Machado, MD, + Gustavo Cordeiro, MD, + \\ and Luís Cunha, $\mathrm{PhD} \dagger$
}

\begin{abstract}
Background: Intracranial atherosclerotic disease is a common cause of stroke; its incidence and prevalence vary widely by ethnicity. The aim of our study was to analyze the recurrence rate of cerebrovascular events in patients with symptomatic and asymptomatic intracranial stenosis (IS). Methods: We conducted a historical cohort study including all patients admitted in our hospital for stroke or transient ischemic attack (TIA) during 2011 and 2012 with information on intracranial circulation (ultrasonography and/or computed tomography angiography). We identified patients with symptomatic and asymptomatic IS and studied the recurrence of cerebrovascular events (TIA or ischemic stroke within the territory of the stenosis) for a minimum follow-up period of 6 months after the diagnosis of IS. For the recurrence rate estimation, patients with other potentially embolic diseases (in cervical arteries or heart) were excluded. We calculated the rate of recurrence of cerebrovascular events and performed Kaplan-Meier survival curves for symptomatic and asymptomatic IS. Results: We investigated 1302 patients, mean age was 72.41 years (standard deviation 12.75). We identified 218 IS in 158 patients, 77 were symptomatic and 141 were asymptomatic. The recurrence rate of cerebrovascular events was 12.32 per 100 patient-years, with a mean time to recurrence of 1.73 months for symptomatic intracranial stenosis (SIS) and .88 per 100 patient-years for asymptomatic IS $(P<.001)$. Conclusions: These results indicate a high risk of early recurrence of stroke in the territory of a SIS, highlighting the importance of its early diagnosis and aggressive treatment. Key Words: Intracranial atherosclerotic disease-intracranial stenosis-recurrence rate-time to recurrence.
\end{abstract}

(C) 2014 by National Stroke Association

Intracranial atherosclerotic disease (ICAD) is characterized by the development, progression, and complication of atherosclerotic lesions affecting large intracranial arteries. ICAD is an important cause of stroke worldwide and is associated with a high risk of recurrent stroke. ${ }^{1}$ Its

From the *Neurology Department, Centro Hospitalar e Universitário de Coimbra, Coimbra; †Stroke Unit, Centro Hospitalar e Universitário de Coimbra, Coimbra; and $\ddagger$ Faculty of Medicine of the University of Coimbra, Coimbra, Portugal.

Received January 20, 2014; revision received February 24, 2014; accepted March 15, 2014.

Address correspondence to Ana Gouveia, MD, Praceta Prof. Mota Pinto, Av. Bissaya Barreto, 3000-075 Coimbra, Portugal. E-mail: anargouveia86@gmail.com.

$1052-3057 / \$$ - see front matter

(C) 2014 by National Stroke Association

http:/ / dx.doi.org/10.1016/j.jstrokecerebrovasdis.2014.03.015 prevalence varies largely by ethnicity, ranging from $8 \%$ $10 \%$ in Caucasians to $50 \%$ in certain Asian populations.

Corresponding data on asymptomatic ICAD is scarce. Population-based studies in Asia using transcranial Doppler ultrasound found an asymptomatic ICAD prevalence of around $6 \%{ }^{2,3}$ In the metropolitan area of Barcelona, a population-based study analyzing moderate to high vascular risk population showed a prevalence of asymptomatic ICAD of $8.6 \%{ }^{4}$

Apart from few large ICAD prospective studies, data on the natural history of ICAD, especially on asymptomatic intracranial stenosis (AIS), is still limited.

Most studies examining the prognosis of ICAD perform an analysis per patient. However, the treating physician needs to make therapeutic decisions on the stroke risk implicated with a specific artery. In particular, the 
decision on invasive therapeutic strategies directed to a specific stenosis would benefit greatly from the knowledge of the risk of recurrence assigned to that stenosis. As such, a stenosis-based analysis would study the risk attributed to each intracranial stenosis (IS), providing valuable information for the selection of individualized therapeutic strategies.

The purpose of this study was to investigate the prognosis of symptomatic intracranial stenosis (SIS) and asymptomatic intracranial stenosis (AIS) due to atherosclerotic disease. We sought to perform a stenosisbased analysis of the recurrence rate of stroke or transient ischemic attack (TIA) in the territory of the affected artery.

\section{Materials and Methods}

We conducted a historical cohort study including all patients admitted in our hospital for stroke or TIA from January 2011 to December 2012. Local ethics committee's approval was obtained. We excluded patients without information on intracranial circulation. We collected baseline characteristics of the population through consultation of patients' clinical records. Hypertension was defined according to the current guidelines of the European Society of Cardiology $y^{5}$ and diabetes according to the recommendations from the World Health Organization and the American Diabetes Association. ${ }^{6}$ Dyslipidemia was considered present when serum lipid concentration was above the near-optimal level recommended by the American Association of Clinical Endocrinologists. ${ }^{7}$ Smoking was considered when the patient was a current smoker or had quit smoking in the past 6 months.

From this cohort we identified patients with IS. IS was presumed as symptomatic when new neurologic signs were related to an area of the brain supplied by the stenotic artery. Asymptomatic IS was defined as stenosis not related to the current neurologic signs and with no old infarction visible in neuroimaging in its respective vascular territory. Patients with other etiologies for IS such as vasculitis, vasospasm, arterial dissection, or moyamoya disease were excluded.

The intracranial circulation was studied in all patients by transcranial color-coded Doppler (TCCD) ultrasonography. In subjects without transtemporal sonographic window and a clinical suspicion of ICAD, a CT angiography was performed.

TCCD ultrasonography was performed using a handheld $3 \mathrm{MHz}$ sector probe (General Electrics Logiq 7, General Electrics Healthcare, USA). The intracranial arteries studied were intracranial internal carotid artery, M1 and M2 segments of middle cerebral artery, A1 segment of anterior cerebral artery, P1 and P2 segments of posterior cerebral artery, basilar artery, and V4 segment of vertebral artery. IS was classified as mild $(<50 \%)$, moderate $(50 \%-$ $80 \%$ ), and high (80\%) grade, according to peak systolic

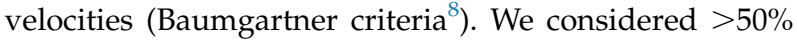
stenosis as clinically significant arterial disease.

In the group of patients with IS, we determined recurrences of cerebrovascular events (TIA or ischemic stroke) within the territory of the previously identified stenosis until June 2013. This information was collected through consultation of patients' clinical records and telephone interviews. We then performed a stenosis-based analysis, calculating the recurrence rate of cerebrovascular events for SIS and AIS. For this analysis, we excluded patients with moderate to severe extracranial artery stenosis tandem to a SIS and patients with known cardiac sources of embolism.

\section{Statistical Analysis}

Comparisons of baseline characteristics were made between patients without IS and patients with SIS and AIS, respectively using independent samples $t$ test (for continuous variables) and $\chi^{2}$ test (for categorical variables).

We performed Kaplan-Meier survival curves for SIS and AIS and compared them using log-rank test. Values of $P<.05$ were considered statistically significant.

\section{Results}

Of the 1540 patients seen in our hospital for cerebrovascular events during 2011 and 2012, we excluded 238 patients without information on intracranial circulation. In the 1302 included patients, we identified 163 patients with IS, corresponding to a total of 240 IS. Excluding nonatheromatous causes of stenosis (3 patients with central nervous system vasculitis, 1 patient with dissection of intracranial vessels, and 1 patient with moyamoya disease), we found 158 patients with IS and 218 IS (77 were symptomatic and 141 were asymptomatic; Fig 1).

Baseline characteristics and vascular risk factors of subjects according to type of IS (symptomatic or asymptomatic) are shown in Table 1 . Patients with AIS were older than patients with SIS $(P=.018)$ and atrial fibrillation (AF) was more frequent in patients with AIS $(P=.032)$.

We calculated a frequency of SIS in our population of $5.76 \%$.

\section{Study of the Recurrences of Cerebrovascular Events}

To perform a stenosis-based analysis of the recurrences, we excluded patients with AF (33 patients) and patients with moderate to severe extracranial stenosis tandem to the IS (8 patients). Thus, for the recurrence analysis, we had 119 patients (corresponding to 171 IS): 72 (60.5\%) with SIS (corresponding to 107 stenosis) and 47 (39.5\%) with AIS (corresponding to 64 stenosis).

In the group of the 171 IS, we had a mean follow-up time of 13.63 months (standard deviation 7.34). We found 15 ischemic events (stroke or TIA) in the territory of the 


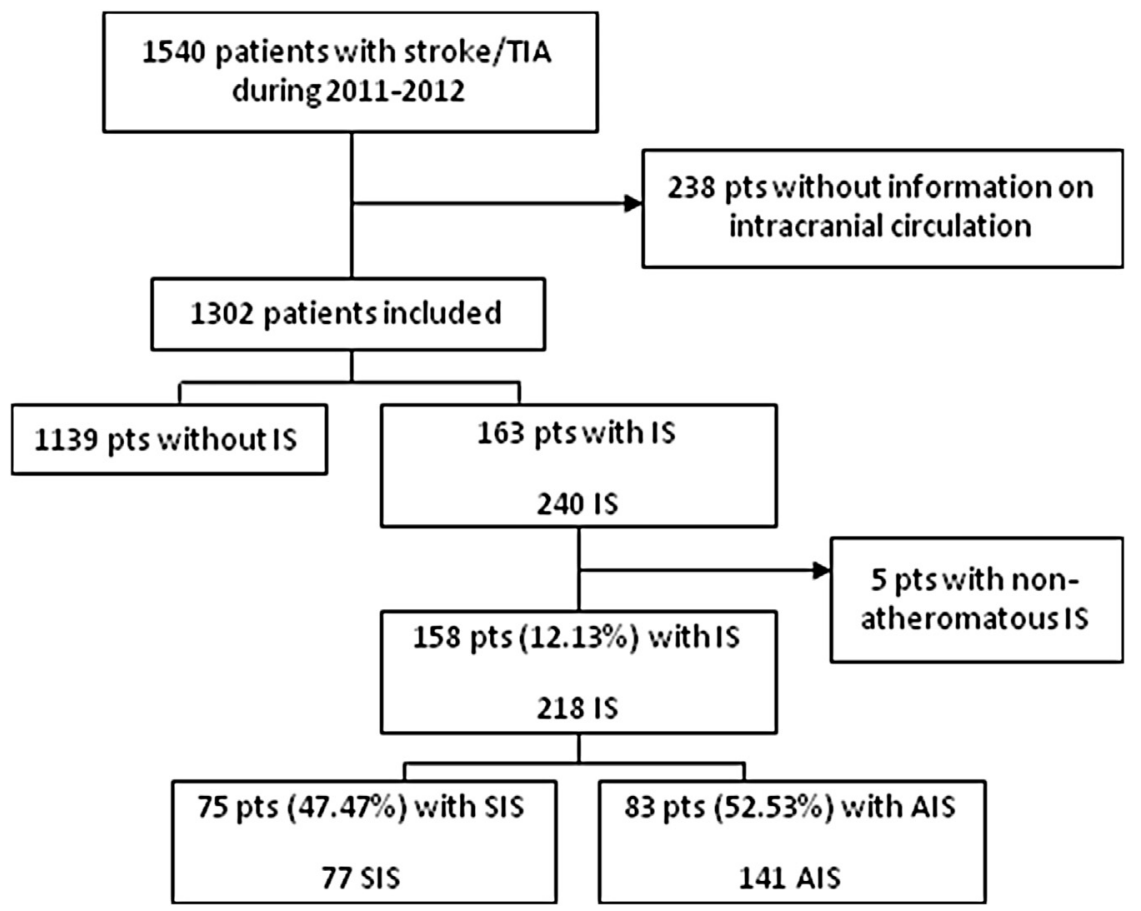

Figure 1. Study design. Abbreviations: AIS, asymptomatic intracranial stenosis; IS, intracranial stenosis; pts, patients; SIS, symptomatic intracranial stenosis.

previously identified stenosis, 14 in SIS and 1 in AIS. We obtained Kaplan-Meier survival curves showing the cumulative probability of cerebrovascular event in the territory of a IS at any time point after baseline for SIS and AIS (Fig 1). The recurrence rate for SIS was significantly higher than for AIS: 12.32 of 100 patient-years versus .88 of 100 patient-years, $P<.001$, with a mean time to recurrence of 1.73 months for SIS.

\section{Discussion}

We have characterized a cohort of 158 patients with ICAD and analyzed the cerebrovascular events recurrence in this group of patients.

Patients with AIS were older and had AF more frequently than patients with SIS. It should be noted that AIS was identified in patients who had an ischemic event not related to IS. Therefore, it is expected that in this group of patients, the proportion of other causes of stroke, namely $\mathrm{AF}$, is higher.

We found a prevalence of SIS in our population of $5.78 \%$, which is slightly lower than the values described for other Caucasian populations. For instance, in the United States, ICAD is responsible for $8 \%-10 \%$ of ischemic strokes in Caucasians. ${ }^{9}$ Nevertheless, as Mazighi et $\mathrm{al}^{10}$ suggested, in a study on autopsies of patients who died as a consequence of ischemic or hemorrhagic stroke, the real impact of ICAD on Caucasians may be greater than expected. According to Asian studies, ICAD accounts for $33 \%-50 \%$ of all strokes in China, $47 \%$ in Thailand, $48 \%$ in Singapore, and 10\%-25\% in Korea. ${ }^{11}$

Table 1. Baseline characteristics of patients and comparison of patients groups with symptomatic and asymptomatic IS

\begin{tabular}{|c|c|c|c|c|c|c|c|}
\hline Baseline characteristics & All patients $(\mathrm{N}=1302)$ & WIS (N = 1139) & $\operatorname{SIS}(N=75)$ & AIS $(\mathrm{N}=83)$ & $P^{*}$ & $P \dagger$ & $P_{\dagger}^{\dagger}$ \\
\hline Age, mean, (SD) & $72.41(12.75)$ & $72.14(12.99)$ & $72.67(10.80)$ & $76.59(9.82)$ & .686 & $<.001$ & .018 \\
\hline Hypertension, n, (\%) & $892(75.1)$ & $755(66.3)$ & $59(78.7)$ & $74(89.2)$ & .413 & .001 & .083 \\
\hline Diabetes, $n,(\%)$ & $325(27.4)$ & $263(23.1)$ & $28(37.3)$ & $32(38.6)$ & .030 & .014 & 1.000 \\
\hline Dyslipidemia, n, (\%) & $505(42.5)$ & $410(40.0)$ & $42(56.0)$ & $51(61.4)$ & .007 & $<.001$ & .520 \\
\hline Smoking, $\mathrm{n},(\%)$ & $109(9.2)$ & $88(7.7)$ & $11(14.7)$ & $9(10.8)$ & .091 & .425 & .484 \\
\hline Atrial fibrillation, $\mathrm{n},(\%)$ & $279(23.5)$ & $246(21.6)$ & $10(13.3)$ & $23(27.7)$ & .034 & .428 & .032 \\
\hline Previous stroke, $\mathrm{n},(\%)$ & $154(13.0)$ & $111(9.7)$ & $24(32.0)$ & $18(21.7)$ & $<.001$ & .007 & .154 \\
\hline
\end{tabular}

Abbreviations: AIS, asymptomatic intracranial stenosis; SIS, symptomatic intracranial stenosis; SD, standard deviation; WIS, without intracranial stenosis.

Values of $p$ with statistical significance are presented in bold typeset.

Patients with nonatheromatous IS were not included in this analysis.

$* P$ value for comparisons between patients without intracranial stenosis and with symptomatic intracranial stenosis.

$\dagger P$ value for comparisons between patients without intracranial stenosis and with asymptomatic intracranial stenosis.

$\ddagger P$ value for comparisons between patients with symptomatic and asymptomatic intracranial stenosis. 


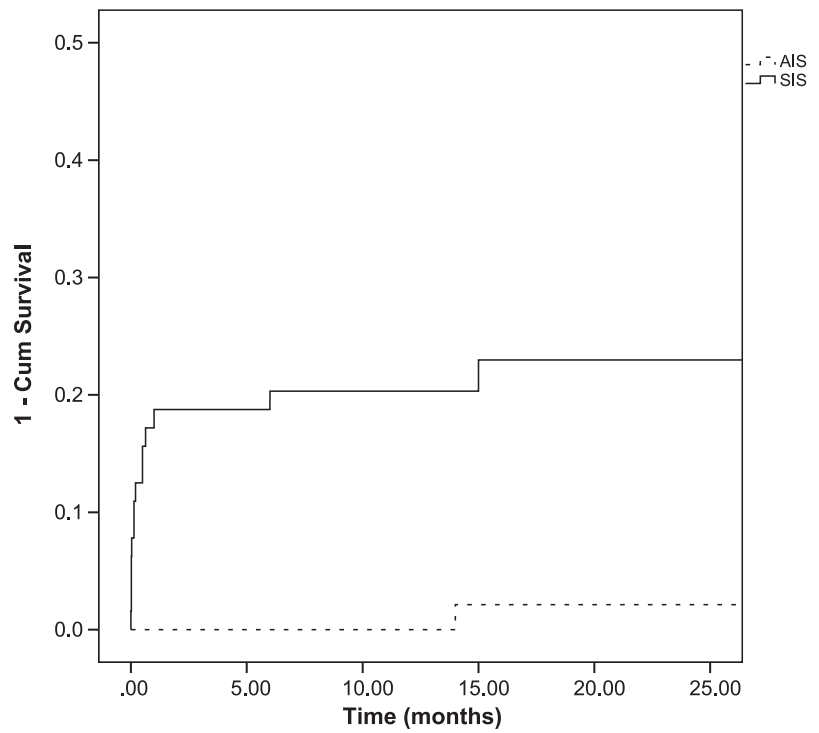

Figure 2. Kaplan-Meier curves for the cumulative probability of an ischemic stroke/TIA in the territory of a stenotic artery versus time. The recurrence rates were 12.32 of 100 patient-years for SIS and .88 of 100 patient-years for AIS, $\mathrm{P}<.001$. Abbreviations: AIS, asymptomatic intracranial stenosis; SIS, symptomatic intracranial stenosis; TIA, transient ischemic attack.

Data on the prognosis of ICAD are scarce, as opposed to extracranial carotid artery disease, where natural history has been extensively studied in several large carotid endarterectomy trials and other prospective studies.

In our population, we found a higher recurrence rate for SIS than for AIS (12.32 per 100 patient-years and .88 per 100 patient-years, respectively). The survival plot (Fig 2) clearly shows that the period of high recurrence risk in SIS is in the first few months after the ischemic event. These data are of extreme importance for the treating physician and is in line with data from previous observational ${ }^{12}$ and randomized studies. In fact, other studies addressing this subject have performed an analysis per patient and their results are close to ours. The largest prospective trial on ICAD compared the natural history of ICAD in patients treated with aspirin and warfarin-the Warfarin versus Aspirin for Symptomatic Intracranial Disease (WASID) trial. ${ }^{13}$ In WASID trial, the annual recurrence rates of stroke in the territory of a stenotic artery were $12 \%$ and $11 \%$ in the aspirin and warfarin arms, respectively. More recently, rigorous data on the influence of endovascular interventions in the natural history of ICAD were provided by the Stenting and Aggressive Medical Management for Preventing Recurrent stroke in Intracranial Stenosis trial. This trial was a randomized controlled trial comparing best medical treatment to best medical treatment plus angioplasty and stent placement (percutaneous transluminal angioplasty and stenting) for symptomatic $>70 \%$ IS. ${ }^{14}$ The global annual recurrence rate of stroke was $14.9 \%$ and $22.3 \%$ in medical therapy and PTAS arms, respectively. ${ }^{15}$

Asymptomatic ICAD has been less well studied, especially in Caucasians. Our current understanding of the prev- alence and natural history of AIS comes, mostly, from studies of patients who have SIS elsewhere or other neurologic conditions, in whom AIS are concomitantly detected. Thus, selection of such patients for the study of the prognosis of asymptomatic ICAD possibly overestimates the risk of cerebrovascular events attributed to AIS. ${ }^{16}$ In WASID trial, AIS was detected in $27.3 \%$ of patients undergoing magnetic resonance angiogram. The annual risk of cerebrovascular events in the territory of the AIS was 3.5\%. ${ }^{17}$ Our data confirm the low recurrence rates attributable to AIS.

As these data suggest, ICAD, in the form of SIS, carries one of the highest rates of stroke recurrency in vascular neurology, with a greater propensity for very early recurrences. Conversely, asymptomatic ICAD appears to represent a relatively benign condition with low annual incidences of stroke and TIA.

To study the effective recurrence rates of IS, we excluded patients with other embolic diseases, namely extracranial tandem disease and cardioembolic emboligenous pathologies, potentially capable of causing ischemic events virtually impossible to distinguish from those caused by IS. We believe this allowed a more accurate estimation of recurrence rates directly attributable to each stenosis.

There are some limitations in our study. First, it is a single-center, historical cohort study. Nonetheless, considering the hard endpoints analyzed it is unlikely to suffer from loss of information. Second, patients' ongoing treatments were not analyzed. This may have led to underestimation of the recurrence rates, especially of SIS. However, it should be noted that all patients were followed in a single tertiary center with homogeneous treatment strategies, thus potentiating an estimation of "real-life" recurrence rates. Moreover, the diagnosis of IS was mostly based in TCCD, which proved to reliably exclude the presence of $50 \%$ $99 \%$ IS, with a negative predictive value of $86 \%$. ${ }^{18} \mathrm{CT}$ angiography was only performed in selected cases of clinical suspicion. As such, some patients $(238,15.45 \%)$ did not have data on intracranial circulation and were excluded. This could have led to a slight underestimation of the prevalence of IS among our population. Notwithstanding, it should also be taken under consideration that these were patients with low level of clinical suspicion of IS; therefore, this limitation is not expected to significantly impair the results obtained.

To conclude, the present study indicates a high risk of early recurrence in patients with SIS, as opposed to AIS, which appears to have a more benign prognosis. Recurrence rates were estimated for each stenosis and excluding other potentially emboligenous diseases; thus, providing more precise information to plan future individualized therapeutic interventions.

\section{References}

1. Gorelick PB, Wong KS, Bae H-J, et al. Large artery intracranial occlusive disease: a large worldwide burden but a relatively neglected frontier. Stroke 2008;39:2396-2399. 
2. Huang HW, Guo MH, Lin RJ, et al. Prevalence and risk factors of middle cerebral artery stenosis in asymptomatic residents in Rongqi County, Guangdong. Cerebrovasc Dis 2007;24:111-115.

3. Wong KS, Huang YN, Yang HB, et al. A door-to-door survey of intracranial atherosclerosis in Liangbei County, China. Neurology 2007;68:2031-2034.

4. Lopez-Cancio E, Dorado L, Millan M, et al. The Barcelona-Asymptomatic Intracranial Atherosclerosis (AsIA) study: prevalence and risk factors. Atherosclerosis 2012;221:221-225.

5. Mancia G, Fagard R, Narkiewicz K, et al. 2013 ESH/ESC guidelines for the management of arterial hypertension: the Task Force for the Management of Arterial Hypertension of the European Society of Hypertension (ESH) and of the European Society of Cardiology (ESC). Eur Heart J 2013;34:2159-2219.

6. Diagnosis and classification of diabetes mellitus. Diabetes Care 2012;35(Suppl 1):S64-S71.

7. Jellinger PS, Smith DA, Mehta AE, et al. American Association of Clinical Endocrinologists' Guidelines for Management of Dyslipidemia and Prevention of Atherosclerosis. Endocr Pract 2012;18(Suppl 1):1-78.

8. Baumgartner RW, Mattle HP, Schroth G. Assessment of $\geq 50 \%$ and $<50 \%$ intracranial stenoses by transcranial color-coded duplex sonography. Stroke 1999;30:87-92.

9. Sacco RL, Kargman DE, Gu Q, et al. Race-ethnicity and determinants of intracranial atherosclerotic cerebral infarction. The Northern Manhattan Stroke Study. Stroke $1995 ; 26: 14-20$.
10. Mazighi M, Labreuche J, Gongora-Rivera F, et al. Autopsy prevalence of intracranial atherosclerosis in patients with fatal stroke. Stroke 2008;39:1142-1147.

11. Wong LKS. Global burden of intracranial atherosclerosis. International J Stroke 2006;1:158-159.

12. Kate M, Sylaja PN, Kesavadas C, et al. Imaging and clinical predictors of unfavorable outcome in medically treated symptomatic intracranial atherosclerotic disease. J Stroke Cerebrovasc Dis 2014;23:973-978.

13. Chimowitz MI, Lynn MJ, Howlett-Smith $\mathrm{H}$, et al. Comparison of warfarin and aspirin for symptomatic intracranial arterial stenosis. N Engl J Med 2005; 352:1305-1316.

14. Chimowitz MI, Lynn MJ, Turan TN, et al. Design of the Stenting and Aggressive Medical Management for Preventing Recurrent Stroke in Intracranial Stenosis trial. J Stroke Cerebrovasc Dis 2011;20:357-368.

15. Chimowitz MI, Lynn MJ, Derdeyn CP, et al. Stenting versus aggressive medical therapy for intracranial arterial stenosis. N Engl J Med 2011;365:993-1003.

16. Taylor RA, Kasner SE. Natural history of asymptomatic intracranial arterial stenosis. J Neuroimaging 2009; 19(Suppl 1):17S-19S.

17. Nahab F, Cotsonis G, Lynn M, et al. Prevalence and prognosis of coexistent asymptomatic intracranial stenosis. Stroke 2008;39:1039-1041.

18. Feldmann E, Wilterdink JL, Kosinski A, et al. The Stroke Outcomes and Neuroimaging of Intracranial Atherosclerosis (SONIA) trial. Neurology 2007;68: 2099-2106. 